\title{
Challenges in prenatal screening and counselling for fragile $\mathrm{X}$ syndrome
}

\author{
Annisa SL Mak, MRCOG, FHKAM (Obstetrics and Gynaecology), KY Leung *, MD, FHKAM (Obstetrics and Gynaecology) \\ Department of Obstetrics and Gynaecology, Queen Elizabeth Hospital, Jordan, Hong Kong \\ *kyleung@ha.org.hk
}

Hong Kong Med J 2017;23:108-9

DOI: $10.12809 / \mathrm{hkmj} 175064$

Fragile X syndrome (FXS) is the most frequent cause of intellectual disability after Down syndrome. It is caused by the expansion of an unstable cysteineguanine-guanine (CGG) trinucleotide repeat on the 5' untranslated region of the fragile $\mathrm{X}$ mental retardation-1 (FMR1) gene. In full mutation (FM), the expansion is $>200$ CGG repeats with aberrant methylation of the promoter region causing loss of the gene expression. Affected individuals display a spectrum of neurological, psychiatric, and developmental problems, as well as abnormal ophthalmological and facial features.

Fragile $\mathrm{X}$ syndrome is an $\mathrm{X}$-linked, dominant disorder. Although all males with a FM have FXS, only half of the females with a FM are clinically affected because of X-chromosome inactivation. The inheritance pattern of FXS is distinctive because a healthy woman who carries a pre-mutation (PM) or a mild expansion of 55-200 CGG repeats can pass on a FM to a child through mitotic expansion of the unstable PM allele. In Cheng et al's article, ${ }^{1}$ two PM carriers (1 in 1325) and one FM carrier (1 in 2650) were detected in a sample of 2650 Hong Kong Chinese pregnant women. In Chinese children with unknown intellectual developmental disorder, the prevalence of FXS has been reported to be $0.93 \% .^{2}$ It would appear that FXS is not rare in the Chinese population.

\section{Prenatal screening}

To predict the birth of a FXS-affected children, PM carriers can be identified through screening. According to the American College of Obstetricians and Gynecologists (ACOG), prenatal screening and genetic counselling for FXS should be offered to women with a family or personal history of FXS, unexplained mental retardation or developmental delay, or premature ovarian insufficiency. ${ }^{3}$ The Society of Obstetricians and Gynaecologists of Canada also suggest that fragile $\mathrm{X}$ testing is indicated in a woman who has at least one male relative with autism, mental retardation, or developmental delay of an unknown aetiology within a three-generation pedigree. ${ }^{4}$ Such screening should also be offered to all women who request it after appropriate genetic counselling. ${ }^{3}$ It is controversial, however, to offer universal screening to all pregnant women. In Hong Kong, this screening is self-financed.

Conventionally, analysis of maternal blood samples by Southern blot is performed to identify PM carriers. Recently, polymerase chain reaction (PCR)-based approaches have enabled more rapid and easy testing, and can be relied upon to characterise CGG repeat size. Nonetheless, amplification of large CG-rich fragments and the study of the methylation pattern can be difficult. Cheng's team used a specific FMR1 PCR-based assay that could detect CGG repeat numbers up to 1000, allowing the identification of PM and FM. ${ }^{1}$ The sensitivity of this PCR test was reported to be high (99\%) and the false-positive rate was approximately $1.3 \%$ although this was probably overestimated. ${ }^{1}$ The cost of the test is US\$44, which is not high. ${ }^{1}$

\section{Genetic counselling}

Although obtaining a maternal blood test for FXS carrier screening is relatively simple, genetic counselling is not, as rightly pointed out by Cheng et al. ${ }^{1}$ In their study, pre-test counselling was given by a research assistant with a bachelor's and master's degree in human genetics, supplemented by written information. ${ }^{1}$ Pre-test counselling for FXS is more difficult than for Down syndrome screening. First, an extensive multigenerational family history must be taken to assess whether FXS, developmental, neurodegenerative, or reproductive disability is present in the mother or any of her family members. Taking such a history is not easy because most patients are not familiar with FXS, and typical phenotypic features of FXS often are not apparent until later childhood. ${ }^{5}$

Second, the inheritance pattern of FXS is complex. Women should be informed about the various outcomes possible and the implications of detecting FM, PM, and intermediate-sized allele (ie 45-54 CGG repeats) results. Prenatal diagnosis should be offered to all pregnant women who are FM or PM carriers. ${ }^{6}$ For a female PM carrier, the risk of expanding to a FM in offspring is dependent on the size of the PM, and is above $98 \%$ for alleles with $>100$ repeats. ${ }^{7}$ For PM carriers with $<69$ repeats, the number of AGG (adenine-guanineguanine) interruptions within the CGG repeat tract (eg occurrence of one AGG interruption after nine uninterrupted CGG repeats is described as: [(CGG)9AGG(CGG)9AGG(CGG)9]) may predict the risk. ${ }^{8}$ The latter risk is higher when there is a positive family history of FXS. 
The prevalence of intermediate-sized allele carriers was shown to be $1.1 \%$ in a local study. ${ }^{1}$ It is difficult to counsel these carriers because the risk for CGG expansion, although very low, is uncertain. Thus, prenatal diagnosis may be offered on a caseby-case basis. Expansion of an intermediate-sized allele into FM may occur in two generations. The European Molecular Genetics Quality Network recommends genetic counselling be offered to family members of intermediate carriers with 50-54 CGG repeats because they may carry a PM. ${ }^{9}$

Prenatal diagnosis can be achieved by determination of CGG expansion and methylation status using a combination of PCR and Southern blot analysis on a sample from chorionic villus sampling (CVS) or amniocentesis. Methylation results from CVS must be interpreted with caution because methylation of a FM is not always present before 14 weeks of gestation, ${ }^{10}$ and the FMR 1 gene is not methylated on the inactive $\mathrm{X}$ chromosome in a female fetus. A follow-up amniocentesis may be required if (a) determination of the methylation status is required to differentiate a large PM from a small FM, or (b) exclusion of somatic mosaicism with FM is required in PM. In all cases, maternal contamination should be excluded, and the gender of the fetus determined to interpret the results of the mutation study.

If a female FM carrier fetus is identified through prenatal diagnosis, there is no way to tell whether the fetus is affected by FXS. Their parents will face uncertainty and anxiety about the resulting phenotype, and have a difficult choice to make. Termination of such pregnancy was reported previously and in the study by Cheng et al. ${ }^{1}$ On the contrary, in the same study, a woman with PM declined prenatal diagnosis owing to the unpredictable phenotype in a FM female. ${ }^{1}$

Pre-mutation carriers are at risk of developing fragile $\mathrm{X}$-associated tremor/ataxia syndrome (FXTAS) and fragile X-associated primary ovarian insufficiency (FXPOI). Onset of FXTAS is typically in the sixth decade of life, and older males are at high risk. ${ }^{11}$ Approximately 20\% of female PM carriers may suffer from early menopause below the age of 40 years, ${ }^{12}$ and thus appropriate reproductive interventions should be informed.

Sufficient time should be allowed for women to review and consider the information given, including the complex inheritance pattern and implications of FXS. Women generally know little about FXS prior to counselling. Adequate psychosocial support should be given to the mutation carriers who may become anxious when they know the uncertain inheritance risk of expansion from PM to FM, the uncertain phenotype of a female FM carrier, or the future risks of FXPOI and FXTAS. Family dynamics must also be considered. Although screening for FXS should be offered to other at-risk family members, such extended or cascade screening may be declined as in
Cheng et al's study. ${ }^{1}$

In summary, ACOG recommends offering prenatal screening and genetic counselling for FXS to all at-risk women. ${ }^{3}$ Risk factors can be identified by taking an extensive multigenerational family history although this may be difficult. The provision of appropriate counselling is a challenge in view of the time and knowledge required to discuss the screening process, the complex inheritance pattern and heterogeneous phenotype of FXS, and its potential impact on psychosocial status and family members. Providing medical education to obstetricians, midwives and genetic counsellors, and targeted education materials to pregnant women may help. ${ }^{13}$

\section{References}

1. Cheng YK, Lin CS, Kwok YK, et al. Identification of fragile $\mathrm{X}$ pre-mutation carriers in the Chinese obstetric population using a robust FMR1 polymerase chain reaction assay: implications for screening and prenatal diagnosis. Hong Kong Med J 2017;23:110-6.

2. Chen X, Wang J, Xie H, et al. Fragile X syndrome screening in Chinese children with unknown intellectual developmental disorder. BMC Pediatr 2015;15:77.

3. American College of Obstetricians and Gynecologists Committee on Genetics. ACOG Committee Opinion No. 469: Carrier screening for fragile X syndrome. Obstet Gynecol 2010;116:1008-10.

4. Genetics Committee of the Society of Obstetricians and Gynaecologists of Canada (SOGC); Prenatal Diagnosis Committee of the Canadian College of Medical Geneticists (CCMG), Chitayat D, et al. Fragile X testing in obstetrics and gynaecology in Canada. J Obstet Gynaecol Can 2008;30:837-46.

5. Bailey DB Jr, Raspa M, Bishop E, Holiday D. No change in the age of diagnosis for fragile $\mathrm{X}$ syndrome: findings from a national parent survey. Pediatrics 2009;124:527-33.

6. Ram KT, Klugman SD. Best practices: antenatal screening for common genetic conditions other than aneuploidy. Curr Opin Obstet Gynecol 2010;22:139-45.

7. Nolin SL, Glicksman A, Ding X, et al. Fragile X analysis of 1112 prenatal samples from 1991 to 2010. Prenat Diagn 2011;31:925-31.

8. Nolin SL, Sah S, Glicksman A, et al. Fragile X AGG analysis provides new risk predictions for 45-69 repeat alleles. Am J Med Genet A 2013;161A:771-8.

9. Biancalana V, Glaeser D, McQuaid S, Steinbach P. EMQN best practice guidelines for the molecular genetic testing and reporting of fragile $\mathrm{X}$ syndrome and other fragile $\mathrm{X}$ associated disorders. Eur J Hum Genet 2015;23:417-25.

10. Devys D, Biancalana V, Rousseau F, Boué J, Mandel JL, Oberlé I. Analysis of full fragile X mutations in fetal tissues and monozygotic twins indicate that abnormal methylation and somatic heterogeneity are established early in development. Am J Med Genet 1992;43:208-16.

11. Jacquemont $S$, Hagerman RJ, Leehey MA, et al. Penetrance of the fragile $\mathrm{X}$-associated tremor/ataxia syndrome in a premutation carrier population. JAMA 2004;291:460-9.

12. Sherman SL. Premature ovarian failure in the fragile $X$ syndrome. Am J Med Genet 2000;97:189-94.

13. Espinel W, Charen K, Huddleston L, Visootsak J, Sherman S. Improving health education for women who carry an FMR1 premutation. J Genet Couns 2016;25:228-38. 